\title{
« Instaurer plus de coopération entre les professionnels de l'Information-Documentation des CCSTI et des Musées »
}

Olivier Soichot et Maryline Blondeau

\section{OpenEdition}

\section{Journals}

Édition électronique

URL : http://journals.openedition.org/ocim/849

DOI : $10.4000 /$ ocim. 849

ISSN : 2108-646X

Éditeur

OCIM

Édition imprimée

Date de publication : 1 mars 2011

Pagination : 38-44

ISSN : 0994-1908

\section{Référence électronique}

Olivier Soichot et Maryline Blondeau, « «Instaurer plus de coopération entre les professionnels de I'Information-Documentation des CCSTI et des Musées » », La Lettre de I'OCIM [En ligne], 134 | 2011 , mis en ligne le 01 mars 2013, consulté le 30 avril 2019. URL : http://journals.openedition.org/ ocim/849; DOI : 10.4000/ocim.849

Ce document a été généré automatiquement le 30 avril 2019

Tous droits réservés 


\title{
"Instaurer plus de coopération entre les professionnels de l'Information- Documentation des CCSTI et des
} Musées »

\author{
Olivier Soichot et Maryline Blondeau
}

Depuis quand le Forum départemental des Sciences dispose-t-il d'un service de documentation?

1 Le Forum des Sciences tel qu'on le connaît dans son lieu et ses missions actuels a été officiellement inauguré le 14 décembre 1996. Pourtant, il a été préfiguré dès 1982 sous la forme d'un CCSTI (association Alias) créé alors par Bernard Maitte. À ce moment là, le centre de documentation avait déjà été imaginé. Il était prévu de faire fonctionner ce service avec sept documentalistes et un objectif prioritaire : offrir un appui aux projets éducatifs et culturels auprès des professionnels de l'animation, de l'enseignement, de la culture ou toute personne porteuse d'un projet de diffusion des sciences et des techniques auprès du grand public.

2 À l'époque, il s'agissait d'une démarche vraiment novatrice. Aujourd'hui, ce fonctionnement orienté autour du public relais/intermédiaire (les professionnels de l'extérieur) reste prépondérant et perdure toujours, même si, à l'instar de ce qui se fait beaucoup plus communément dans les autres CCSTI, l'appui aux projets des services en interne est important.

3 Par ailleurs, il faut noter que, si depuis 2006, le Forum départemental des Sciences est devenu un équipement du Conseil général du Nord, ce statut particulier n'a pas influé directement sur le fonctionnement de son service documentaire, hormis en termes de gestion administrative et budgétaire (marché abonnements ou acquisitions par exemple... ) . 
Aujourd'hui, en quoi consiste exactement l'activité documentaire au sein de votre structure?

Malgré les aléas financiers que nous avons subis avant la départementalisation en tant qu'association, le service de documentation s'est toujours efforcé de maintenir son cœur de métier, c'est-à-dire l'appui aux projets éducatifs et culturels. Aujourd'hui, nos principaux usagers extérieurs restent les professionnels de l'animation, de l'enseignement, de la culture, des collectivités... Pour autant, on a observé une évolution au niveau de ces différentes catégories d'acteurs. Ainsi, il y a une dizaine d'années, 60 à $70 \%$ d'entre eux étaient liés à l'enseignement contre $40 \%$ aujourd'hui (principalement des écoles et des collèges). Actuellement, les professionnels hors Éducation (de la culture, de l'animation...) représentent la majorité de nos usagers, dont beaucoup sont issus d'associations culturelles (les compagnies de théatre par exemple) et surtout, depuis quelques années, des collectivités territoriales au travers de leurs différents services " jeunesse ", « animation "..., des Unités Territoriales de Prévention et d'Action Sociale (UTPAS). Peu de temps après ma prise de fonction en 2004, je suis allée à la rencontre de ces acteurs pour essayer d'avoir une politique offensive vers ces publics potentiels du « centre de doc ». Cela leur a sans doute permis de se rapprocher un peu plus de l'offre de notre activité. L'évolution de leur structuration interne avec la mise en place de secteurs « jeunesse/petite enfance », « environnement » ou encore « développement durable » les a sans doute aussi incités à nous fréquenter davantage.

Avec un spectre d'usagers aussi large, comment s'organise un service de documentation comme le vôtre?

5 À mon arrivée, j'ai découvert un fonds extrêmement riche avec des documents fictions et documentaires pour tous types de public, sur la culture scientifique, technique et industrielle. En termes de centres d'intérêts de nos usagers, les thématiques liées à la santé (pour les secteurs jeunesse, les UTPAS, les Instituts Médico-Éducatifs...) fonctionnent très bien, tout comme celles renvoyant à l'environnement, voire à la technologie (intérêt très fort des lycées professionnels). Pour répondre au mieux aux besoins des structures, on commence par cibler et sélectionner nos propres ressources (documentaires et de découvertes scientifiques), puis celles de l'extérieur, qu'il s'agisse de personnes ressources comme des conférenciers, des vulgarisateurs ou médiateurs ou "d'outils éducatifs» tels que malles ou ateliers pédagogiques... ou encore d'événementiels (expositions, séances théâtre, animations). L'aide aux projets peut s'accompagner ou non d'emprunt de nos ressources documentaires. Car, de fait, on assure, comme tout centre de documentation, le prêt (gratuit) de documents. Les prêts se font essentiellement sur les thématiques précitées mais aussi vers des rayons plus classiques comme celui touchant à l'astronomie, aux sciences générales, ou aux expériences par exemple. Outre ceux bénéficiant de notre appui documentaire à leur projet, il y a aussi des publics de professionnels, très réguliers, qui font leur sélection de ressources eux-mêmes; dans les deux cas, nous sommes vraiment "médiateurs" de ressources (sélection et présentation d'ouvrages/ressources correspondant vraiment aux besoins formulés par le professionnel pour un public particulier de manière à ce qu'il dispose d'une documentation pertinente). Nous prêtons également des vidéos dont nous achetons les droits pour que notre public de professionnels puisse les diffuser auprès de son propre public...

6 En ce qui concerne l'appui aux projets internes, d'une part nous apportons notre connaissance des éditions et collections scientifiques; ce, pour cibler les ouvrages ajustés 
à la préparation d'une création d'exposition / d'un scénario d'animation, ou à la réalisation d'espaces lecture grand public intégrés dans chaque exposition (petits 3-6 ans, + de 7 ans et ados-adultes); d'autre part, notre valeur ajoutée s'exerce incontestablement par le traitement de la presse quotidienne et des magazines de vulgarisation scientifique qui servent d'appui pour défricher et situer une thématique d'exposition ou pour réaliser nos propres produits documentaires d'actualité (revue de presse ou panneaux de presse) mettant en perspective le sujet de l'exposition ou d'un événement...

Combien de personnes sont rattachées au service documentation?

7 L'équipe se compose actuellement de cinq personnes : trois documentalistes généralistes, assurant le traitement du document et de l'information et référente chacune en interne sur l'appui documentaire à un projet d'exposition; une documentaliste assistante, spécialisée sur l'aspect documentation/recherche d'images et droits afférents et qui se positionne aussi sur l'aspect administratif du service (abonnements, commandes, suivi budgétaire...) ; et une employée de documentation, directement en charge de l'accueil et orientation des publics, de la gestion des prêts, des collections...

Concrètement et à travers quelques exemples, comment s'articule aujourd'hui le fonctionnement de l'activité documentaire avec celui du CCSTI ?

8 Le contexte particulier de création du centre de documentation a, au départ, largement influé sur son activité. Ainsi, au début, celle-ci était orientée, à part égale entre l'appui documentaire pour l'interne et pour l'externe. Depuis 2000, une amorce s'est progressivement opérée pour venir davantage en appui interne aux services du Forum en raison de certains événements propres à son fonctionnement. Par exemple, il existait auparavant un service « Actualités » dont le responsable travaillait en liaison étroite avec la Doc, en exploitant le dépouillement de la presse fait par les documentalistes pour provoquer l'organisation de débats ou développer de nouveaux thèmes d'expositions. À partir de 2004, ce service a cessé de fonctionner et à mon arrivée, la direction m'a demandé de le redéployer sous une autre forme (de type veille interne) au sein du service. De fait, l'activité pour l'extérieur a été forcément réduite.

9 A contrario, il y a eu des périodes où l'on travaillait moins en interne car la situation financière était peu propice à la création. Les services internes ayant moins besoin de la Doc, nous en profitions pour avoir une démarche plus offensive à l'extérieur ou pour travailler sur des missions plus à la marge comme la critique de l'édition scientifique. Aujourd'hui, la répartition entre notre activité interne et externe a retrouvé son équilibre initial, même si cette articulation particulière n'est pas toujours évidente. Certaines tâches documentaires, destinées au fonctionnement propre au centre de documentation passent parfois au second plan au profit d'autres, davantage utiles au niveau global pour le Forum. Le rattachement de la Doc au sein d'un CCSTI, fait qu'on va forcément donner la priorité à l'appui aux expositions ainsi qu'aux actualités en interne, plutôt qu'au traitement de documents dont le thème n'est pas directement lié aux expositions par exemple... Enfin, faisant partie du Pôle Programmation/Création du Forum, nous travaillons en mode projet, ce qui signifie que les documentalistes comme tout participant à un groupe projet transversal, sont tributaires du cadre et des délais impartis pour celui-ci.

En plus d'être une professionnelle de la documentation, vous êtes également attachée de conservation. Vous avez donc un regard privilégié sur les liens qui existent entre le monde 
des musées et celui de l'activité documentaire en CSTI. Justement, comment voyez-vous aujourd'hui le lien entre ces deux champs particuliers?

10 De par mes choix professionnels, le terme «conservation" me touche en effet particulièrement. Aussi, je dirais que le lien entre le monde des musées et celui de la documentation me paraît actuellement assez lointain ou tout du moins très léger. En fait, hormis à l'OCIM, je n'observe pas vraiment de proximité clairement affichée entre ces deux secteurs. Bien sûr, on retrouve entre eux un vocabulaire commun comme par exemple les termes "collection", " conservation », " acquisition", mais ces mots renvoient plutôt à une notion documentaire au sens biblio-économique. Dans un CCSTI, on ne retrouve pas la notion de conservation pure au sens où l'entend un musée. Et pour cause, nous n'avons pas de collections c'est-à-dire d'objets. Nous n'avons même pas d'objectifs de conservation, y compris pour ce que l'on pourrait appeler «les dossiers d'expositions ». Naturellement, dans le cadre de notre activité documentaire, nous pouvons être amenés à conserver des fonds iconographiques mais cette démarche s'inscrit dans une durée limitée qui correspond au temps de l'exposition, ou de son itinérance.

Dans ce cas, un documentaliste de musée est donc profondément différent d'un professionnel de la documentation travaillant dans un CCSTI?

11 Je répondrais à la fois oui et non. Non, si on parle du métier de documentaliste et des techniques documentaires pures: traitement du document, maîtrise de l'informatique documentaire et de la recherche documentaire, réalisation de produits documentaires, bibliographies...

12 Mais oui, si on parle des activités propres aux structures dans lesquelles le documentaliste s'insère. Par exemple, une des fonctions inhérentes au documentaliste de musée concerne les dossiers d'œuvre (ndlr : appelés aussi dossiers de collection ou dossiers d'acquisition), qui font référence aux objets d'un musée et caractérisent chacune des pièces acquises, conservées ou exposées au musée. Ces dossiers sont donc propres au centre de documentation du musée et vont être conservés sur une échelle de temps qui n'est pas du tout la nôtre en CCSTI.

13 Comme tout centre de documentation, notre seul objectif de conservation porte sur les collections de documents ou de périodiques. A contrario, nous avons en CCSTI une mission assez spécifique de traitement de l'actualité scientifique (dépouillement de l'information presse) qui peut se concrétiser par différents moyens (revues de presse, rédaction de brèves d'actualité...) : il me semble que cette mission n'est pas impérative pour un documentaliste de musée...

Comment ressentez-vous les relations entre conservateurs et professionnels de la documentation?

14 Pour ma part, je côtoie beaucoup de conservateurs et lorsque je me présente comme professionnelle de la documentation, la plupart d'entre eux ne font pas du tout le lien entre leur activité et la mienne. Mis à part les conservateurs des archives départementales, il faut vraiment que j'adopte le vocabulaire propre à la muséologie pour qu'ils soient plus attentifs. Le fait d'être également attachée de conservation m'aide heureusement à faire ce lien plus facilement. Cela dit, il existe encore très peu de passerelles entre nos deux mondes pour nous permettre de mieux nous comprendre et donc de mieux travailler ensemble. Ce manque d'articulation entre nos différentes activités m'interpelle. Je souhaiterais qu'il y ait davantage de coopération entre les professionnels de l'Information-Documentation en CCSTI et ceux des musées. Chacun a à 
y gagner. L'aspect « science et société », censé être développé en CCSTI, en serait enrichi : car on ne peut comprendre les enjeux sociétaux des découvertes scientifiques sans avoir un peu de recul et de regard sur le passé... Il faut donc se décloisonner et s'ouvrir à la différence pour en faire une complémentarité. Cela vaut, à mon sens, pour les documentalistes comme pour les médiateurs, animateurs des deux côtés (CCSTI et musées). Je crois que certains pays comme l'Italie ou l'Angleterre sont un peu plus en avance que nous en la matière. Ils essaient justement de créer des ponts entre l'aspect conservation et l'aspect actualité ; entre le passé et le futur.

Sous quelles formes pourrait être envisagée cette coopération dont vous parlez?

Je trouve que la valorisation d'un objet telle qu'on peut la concevoir dans un musée pourrait être envisagée sous un angle résolument coopératif. On pourrait par exemple imaginer l'emprunt d'un objet de musée pour le valoriser au sein d'un CCSTI. Pour nous, CCSTI, cela nous permettrait de mieux développer le volet "Technique » et "Industrielle» de la culture scientifique telle que ce type de structure cherche à le promouvoir. À l'inverse, le centre de documentation d'un CCSTI pourrait collaborer à la valorisation d'une œuvre d'un musée en proposant du contenu additionnel en lien notamment avec l'actualité scientifique. Par exemple, sous forme de panneaux de presse ou de revues de presse pour contextualiser davantage un objet muséal. Il me semble que cela commence à se faire en France, au travers de petites expositions temporaires du type de celles du musée des Arts et Métiers, sur les trains ou les jeux vidéo par exemple.

L'irruption des Nouvelles Technologies de I'Information et de la Communication a eu une répercussion directe sur les modes d'accès et de diffusion de l'information, ainsi que sur les attentes des publics. De fait, ces technologies numériques ont donc modifié en profondeur l'activité des services de documentation. Pour vous, quels changements principaux ces bouleversements ont-ils impliqué sur les pratiques documentaires en général ?

16 Soulignons d'abord que nous vivons ces changements en permanence! Aujourd'hui, grâce à Internet, tout le monde a accès à l'information. Aussi, il faut sans cesse se repositionner pour valoriser notre activité. Cela passe par un travail de veille quotidien sur les nouveaux outils, les nouvelles pratiques. Je constate que mes homologues basés sur Paris font ça très bien. C'est peut-être moins évident dans des structures de province en raison du clivage qui existe parfois de manière beaucoup plus marquée entre les responsables du service de documentation, les webmasters, les responsables éditoriaux... À nous de montrer nos compétences pour mieux nous affirmer dans l'usage de ces nouvelles technologies dont le potentiel pour notre activité est extraordinaire.

Concrètement, comment appréhendez-vous ces nouvelles opportunités dans votre travail au Forum départemental des Sciences?

17 Malheureusement, nous n'avons pas eu la possibilité de créer un réseau intranet / extranet documentaire tel que nous l'aurions souhaité. Pour l'heure, nous fonctionnons classiquement avec des bases de données pour le traitement des documents mais aussi pour le traitement de l'information avec des logiciels de cartographie qui nous permettent de valoriser notre appui aux projets internes et externes. Ainsi, on va, par exemple modéliser sous forme de carte toutes les informations recueillies sur un concept et défricher ainsi un sujet voué à être mis en exposition. Pour se les approprier pleinement, ces nouveaux outils demandent naturellement un temps d'adaptation et l'acquisition de certaines connaissances techniques. C'est ce que j'essaye de mettre en place petit à petit avec mon équipe et je dois dire que ces nouveautés sont bien accueillies au niveau des documentalistes. 
On le voit, les Nouvelles Technologies de l'Information et de la Communication placent les professionnels de la documentation au carrefour de plusieurs disciplines. Ainsi, on assiste presque à une redéfinition du métier de documentaliste avec un volet éditorial de production et de diffusion de l'information à destination de différents publics. Cette évolution ne produit-elle pas un effet de concurrence avec d'autres activités ?

Avec l'arrivée des technologies numériques, c'est vrai que le management de l'information est devenu un champ dans lequel peuvent s'impliquer pratiquement tous les services d'une même structure. En un sens, ce phénomène a donc placé l'activité documentaire dans un contexte de concurrence par rapport à d'autres secteurs comme la communication, l'édition, la veille ou encore la formation. Pour autant, je n'éprouve personnellement aucune difficulté à me positionner ou à me définir par rapport à ces autres champs professionnels. Je pense que tous les corps de métier ayant un lien plus ou moins fort avec le domaine de l'information peuvent très bien vivre ensemble à partir du moment où ils se respectent. Un responsable éditorial pourra par exemple nous aider à la mise en forme d'une revue de presse ou la rédaction d'un éditorial. De son côté, un profes sionnel de la communication nous apportera son savoir-faire sur la meilleure façon de la diffuser... Les responsables de l'Information-Documentation doivent avoir suffisamment confiance en eux et ne surtout pas se recroqueviller sur eux-mêmes pour s'affirmer à côté de ces différents métiers.

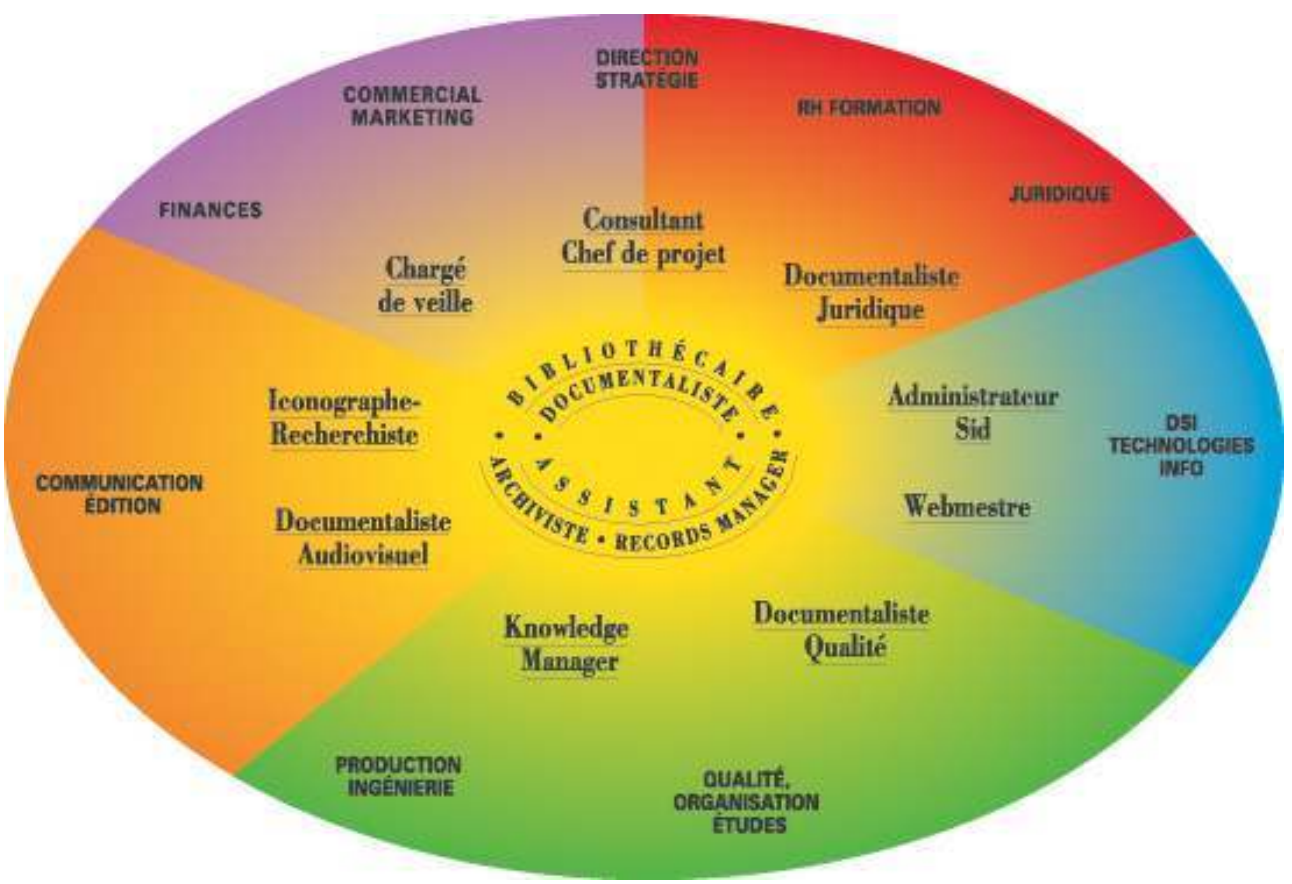

L'Information-Documentation aujourd'hui : une pluralité de métiers au carrefour de différents domaines

(c) Le référentiel des métiers et fonctions, Association des professionnels de l'information et de la documentation, ADBS

Au-delà de l'amélioration de la gestion et de l'accès aux informations, les technologies de l'information et de la communication favorisent également la coopération entre les structures culturelles et donc, leurs services de documentation. Depuis 3 ans, vous participez d'ailleurs activement à un réseau professionnel redynamisé par l'OCIM (voir encadré en annexes). Concrètement que vous apporte ce travail en réseau dans votre activité ?

C'est d'abord l'occasion d'échanger sur nos pratiques professionnelles, de voir quels outils chacun utilise pour éventuellement se les approprier soi-même. Le fait d'appartenir à 
différents types de structures représentant différents corps de métier est d'ailleurs ex trêmement intéressant, notamment dans la perspective de réduire la distance que l'on peut constater entre le monde des musées et celui des CCSTI. Par ailleurs, la mise en place du Wiki nous pousse à utiliser un outil un peu nouveau pour essayer de valoriser le réseau.

Peut-on vraiment imaginer mettre en place un jour une politique documentaire et une gestion de l'information commune dans le champ très large de la CSTI-muséologie?

Parler de gestion commune peut faire peur car il faut bien le reconnaître, les documentalistes font parfois preuve un peu d'individualisme. Tout du moins, beaucoup vivent un peu repliés sur eux-mêmes. C'est justement cet isolement que le réseau cherche à éviter en confrontant les points de vue et les modalités d'exercices. Rassembler tous ces professionnels n'a rien d'évident. Leur trouver un nom commun l'est encore moins. S'il n'y a pas de volonté de se fédérer au niveau national, ce chantier d'une politique documentaire dans le domaine de la CSTI me paraît compliqué à mener.

Pour vous, quelles sont les principales difficultés auxquelles achoppe encore le secteur de l'information documentation au sein des structures culturelles? Peut-on parler d'un manque de reconnaissance des professionnels?

21 Dans le cas du Forum départemental des Sciences comme structure en tant que telle, on ne peut pas parler d'un manque de reconnaissance dans la mesure où, dans la réflexion qui a prévalu à la création du CCSTI, les problématiques liées à la politique documentaire ont été largement intégrées au projet. Par contre, il peut arriver que les différentes directions soient plus ou moins sensibilisées à la réalité de notre activité ${ }^{1}$. Certains notamment nous perçoivent plutôt comme des bibliothécaires. Ils comprennent mal pourquoi il faudrait passer par le service de documentation pour obtenir de l'information alors qu'ils peuvent légitimement la collecter sur leur propre ordinateur. Pour contrer ce regard un peu étriqué posé sur notre profession, à nous d'aller au devant d'eux pour leur expliquer nos missions et surtout, susciter de nouveaux besoins dans les domaines de l'Information-Documentation.

Enfin, quels sont pour vous les grands défis que doit relever votre champ professionnel dans les années à venir?

C'est d'abord aux directeurs que revient le rôle d'impulser une vraie dynamique au niveau de la documentation, notamment dans le domaine de la recherche et de l'exploitation d'informations. Peut-être en redéfinissant leur service d'Information-Docu mentation dans une position plus claire voire stratégique, au niveau de l'organisation de leur structure respective. Mais je le répète, à nous de savoir aussi pratiquer une forme de marketing documentaire pour montrer l'importance de notre activité auprès de nos différents publics et imaginer de nouveaux services. Nous devons être les premiers acteurs de notre existence propre notamment en montrant que nous savons sélectionner des sources d'informations toujours plus nombreuses mais aussi en produisant du contenu informationnel utile. Nous disposons de vraies compétences dans ces deux domaines mais souvent insuffisamment exploitées, notamment dans les musées. Au-delà de la gestion de la chaîne documentaire, nous ne devons pas non plus hésiter à nous former encore plus dans la maîtrise des nouvelles technologies, mais toujours en les utilisant de manière adaptée. On peut par exemple imaginer de les utiliser pour valoriser davantage le patrimoine info-documentaire interne de nos structures, dont on s'aperçoit de plus en plus qu'il présente un réel intérêt stratégique pour les établissements. Pour ré sumer, faisons preuve d'initiative et de créativité pour proposer de nouvelles solutions 
documentaires. Et toujours dans un esprit de service à destination de nos différents publics.

\section{ANNEXES}

\section{Des professionnels regroupés en réseau}

Depuis plus de 15 ans existe un réseau à vocation nationale rassemblant des professionnels de l'Information-Documentation dans les domaines de la muséologie, du patrimoine et de la culture scientifiques, techniques et industriels. Comptant actuellement une dizaine de personnes issues de différentes structures, sa composition se veut représentative de la diversité des champs qu'il couvre.

\section{Une volonté d'échanger}

C'est en 1993 et sous l'impulsion notamment de Carole Braéckman du Centre Régional de Promotion de la Culture Scientifique, Technique et Industrielle de Villeneuve d'Ascq et d'Armelle Pilon, du CCSTI de l'ABRET (Pleumeur-Bodou), que les bases d'un premier réseau informel sont jetées. À l'époque, l'initiative part d'un objectif assez simple : permettre aux professionnels du secteur d'échanger autour des problématiques liées à l'Information-Documentation dans le champ de la culture scientifique et technique. Pour les acteurs, c'est aussi un moyen d'affirmer une identité collective et de combler un manque dans le domaine de la formation des documentalistes de la CSTI. Au début, cette petite communauté de pratiques est animée par l'OCIM (Christelle Détourbet) et se compose d'une poignée de partenaires issus principalement des CCSTI. Assez rapidement et avec l'intégration du musée des Arts et Métiers représenté par Anne Chanteux, le maillage s'étend ensuite aux musées techniques ainsi qu'aux muséums. Avec cette ouverture, l'idée est de reconnaître des similarités de fonctionnement dans l'activité documentaire de différentes catégories de structures. Réunions annuelles, ateliers thématiques (notamment sur le thème des NTIC), échanges d'informations par liste de diffusion, édition d'un bulletin traitant de la documentation dans la CSTI, au fil des ans, le réseau se structure autour de plusieurs actions concrètes. Durant ces premières années, la création d'un portail de « la documentation dans la CSTI et dans les musées » commence aussi peu à peu à être évoquée. Malgré ses efforts, le réseau finira pourtant par s'essouffler en $2006 \ldots$ pour mieux rebondir deux ans plus tard.

\section{Vers une valorisation des ressources documentaires}

En 2008, le réseau décide de repartir sur une nouvelle dynamique en s'appuyant sur un nouveau groupe de travail. Sous l'impulsion de l'OCIM se réunissent alors autour d'une même table des professionnels de plusieurs structures : le musée des Arts et Métiers, la Cité des Sciences et de l'Industrie, le Palais de la Découverte, le service des Musées de 
France, le Forum départemental des Sciences de Villeneuve d'Ascq et l'Institut National du Patrimoine. Fort de ces nouvelles bases, le réseau cible aussi un nouvel objectif. Dans un domaine en recherche de structuration, la construction d'une politique documentaire et de gestion de l'information complémentaire apparaît comme un projet pertinent et indispensable. Ainsi, sans se départir de sa volonté d'échanges d'idées et d'initiatives, le réseau concentre désormais ses efforts pour offrir aux publics et aux acteurs des différents champs qu'il couvre un service de qualité. Ce travail passe notamment par la mise en visibilité des ressources documentaires et de l'information détenue par chacun de ses membres. Cumulés, ces fonds représentent aujourd'hui des centaines de milliers de notices bibliographiques (monographies, titres de périodiques, documents multimédias, bases de données spécialisées), ainsi que des flux multiples d'informations gérés au quotidien. Bref, un véritable « trésor de guerre ». Ainsi, pour centraliser l'ensemble de ces ressources, l'idée de créer un portail documentaire thématique figure plus que jamais au rang des priorités. En attendant de concrétiser cet ambitieux chantier, le réseau veut continuer de grandir pour mieux fédérer dans son ensemble le monde de l'Information et de la Documentation dans le domaine des musées, du patrimoine et de la culture scientifiques et techniques. Mise en place en 2009 par l'OCIM, une plate-forme de travail collaboratif permet déjà de rendre compte de la richesse des échanges engagés par le groupe de travail. Une réflexion passionnante à laquelle devrait prochainement prendre part d'autres acteurs de référence comme le Muséum national d'Histoire naturelle.

(Pour plus d'informations sur le réseau, contacter Stéphane Chevalier, OCIM stephane.chevalier@u-bourgogne.fr, téléphone + 333805898 38)

\section{NOTES}

1. Selon une enquête menée en 2010 par l'association des professionnels de l'information et de la documentation (ADBS), $63 \%$ des professionnels considèrent que leur activité est reconnue au sein de leur entreprise. Enquête métiers salaire 2010, ADBS: www.adbs.fr/servlet/ com.univ.collaboratif.utils.LectureFichiergw ?CODE_FICHIER $=1296567223967$ \&ID_FICHE $=8879$

\section{RÉSUMÉS}

Conservation, collection, inventaire, catalogue, mise en visibilité, sélection, acquisition, dépôt... De prime abord, le champ des musées et celui de la documentation partagent un vocabulaire commun. Au-delà de leurs similitudes lexicales, ces deux mondes se rejoignent aussi dans leurs missions de diffusion et de valorisation de l'information scientifique et technique.

Mais qu'en est-il réellement de cette proximité manifeste entre ces deux domaines ? Responsable de l'activité documentaire au Forum départemental des Sciences de Villeneuve d'Ascq et également attachée de conservation, Maryline Blondeau nous éclaire sur son activité avant de porter son double regard sur la relation entre musée et activité documentaire. 
INDEX

Mots-clés : documentaliste, Forum des Sciences, information-documentation, métier

\section{AUTEURS}

OLIVIER SOICHOT

Olivier Soichot est chargé d'édition à l'OCIM. olivier.soichot@u-bourgogne.fr

\section{MARYLINE BLONDEAU}

Maryline Blondeau est responsable du service de documentation du Forum des Sciences de Villeneuve d'Ascq. tél + 333201936 23, maryline.blondeau@cg59.fr 